\title{
RECHTSINFORMATIK: VON DER ORCHIDEE ZUR MAGERWIESE
}

\author{
Rolf H. Weber
}

\begin{abstract}
Legal Informatics ist heute ein zentrales Themenfeld in der Rechtswissenschaft mit steigender Beachtung in Wissenschaft und Praxis. Die traditionelle Rechtsinformatik hat sich zwar nach Entstehung dieses interdisiplinären Ansatzes nur relativ langsam entwickelt, doch bewirkt der starke Technologieschub der letzten 25 Jahre zwischenzeitlich eine erhebliche, von ERICH SCHWEIGHOFER und dem jährlichen IRISSymposium massgeblich mitgetragene Umgestaltung und zugleich Bereicherung der Rechtslandschaft.
\end{abstract}

\section{Inhaltsverzeichnis}

A. Einleitung..........................................................................................................................6

B. Phänomenologischer Rückblick auf die Ursprünge der Rechtsinformatik................7

I. Geburtsstunde: Computer und Recht ..........................................................................

1. Computer als neue «Maschinen» .......................................................................

2. Frühe Weiterentwicklungen...........................................................................9

II. Paradigmenwechsel in den letzten 25 Jahren ......................................................10

1. Veränderungen durch neue Technologien ......................................................10

2. Erweiterungen der inhaltlichen Themenpalette...........................................11

C. Legal Informatics als Konsequenz des neuen Technologieschubs .............................13

I. Knowhow-Management und -Sharing in der Rechtsberatung...........................13

Prof. Dr. Rolf H. WeBER, em. Professor für Wirtschaftsrecht an der Universität Zürich, daselbst Co-Leiter des Center for Information Technology, Society, and Law, und Rechtsanwalt in Zürich (Bratschi AG). Simon HENSELER, MLaw, Rechtsanwalt, Universität Zürich, danke ich für die wertvolle Unterstützung bei der Vorbereitung dieses Beitrages. 
II. Zugang zu und «Verwaltung» von Wissen ......................................................... 16



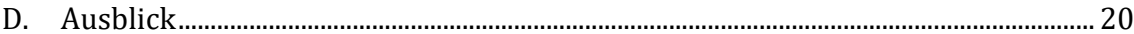

\section{A. EINLEITUNG}

Die modernen Technologien, insbesondere die Digitalisierung und die Distributed Ledger Infrastruktur in all ihren Facetten, prägen das persönliche, berufliche und öffentliche Leben in immer stärkerem Ausmasse. Die Rechtsinformatik («Legal Informatics») ist aber ein Thema, das schon 50 Jahre alt ist; ERICH SCHWEIGHOFER gehört zu den besonders aktiven und einflussreichen Promotoren dieser Rechtsdisziplin. Bereits seine Dissertation befasst sich mit den «Information Retrieval-Systemen» $(1995)^{1}$ und seine Habilitation geht verschiedenen Aspekten der automatischen Textanalyse (1996)2 mit Blick auf die Rechtsinformatik als ein Instrument der Wissensrepräsentation nach.

Die Entwicklungschancen im Lichte der neuen Informationstechnologien und insbesondere die Einsatzmöglichkeiten neuer digitaler «Werkzeuge» haben schon und werden künftig vermehrt wichtige Veränderungen im Kontext der rechtsrelevanten Tätigkeiten zur Folge haben. Als Elemente solcher Entwicklungen lassen sich nennen: ${ }^{3}$

- Einsatz von algorithmischer Software bei der Rechtsausübung (künstliche Intelligenz, Machine Learning);

- Ausbau der Technologien, welche den Umgang mit oder den Zugang zu Recht automatisieren, erleichtern und erheblich verändern;

1 ERICH SCHWEIghofER, Wissensrepräsentation in Information Retrieval-Systemen am Beispiel des EU-Rechts, Diss. Wien 1995, Drucklegung in erweiterter Form, Wien 2000.

2 ERICH SCHWEIGHOFER, Rechtsinformatik und Wissensrepräsentation, Automatische Textanalyse im Völkerrecht und Europarecht, Habil. Wien 1996, publiziert Wien 1999.

3 Rolf H. WeBer, Digitalisierung und der Kampf ums Recht, in: Dal Molin-Kränzlin/Schneuwly/ Stojanovic (Hrsg.), Digitalisierung - Gesellschaft - Recht, Analysen und Perspektiven von Assentierenden des Rechtswissenschaftlichen Instituts der Universität Zürich, Zürich 2019, 3, $8 \mathrm{f}$. 
- Verwendung von Datenverarbeitungsmethoden, die das Rechtssystem, die Rechtsberatung, die Rechtsdienste und die juristischen Arbeitsprozesse unterstützen oder ganz automatisch durchführen.

Diese Entwicklungen hat ERICH SCHWEIGHOFER nicht nur mit seinen eigenen Schriften begleitet, sondern seit über 20 Jahren durch das Internationale Rechtsinformatiksymposion (IRIS) tatkräftig unterstützt; das jährlich durchgeführte IRIS vereinigt an der Rechtsinformatik interessierte Wissenschaftler und Praktiker und ermöglicht dadurch den Austausch von Gedanken zur Weiterentwicklung der Disziplin. ${ }^{4}$

Der vorliegende Beitrag zu Ehren von ERICH SCHWEIGHOFER versucht aufzuzeigen, wie sich die Rechtsinformatik in den letzten 50 Jahren von einer zwar schönen, aber seltenen Orchidee zu einer Magerwiese, d.h. dem artenreichsten Lebensraum mit vielen farbigen Blumen, entwickelt hat.

\section{B. PHÄNOMENOLOGISCHER RÜCKBLICK AUF DIE URSPRÜNGE DER RECHTSINFORMATIK}

\section{Geburtsstunde: Computer und Recht}

\section{Computer als neue «Maschinen»}

Die Herstellung der ersten Computer geht bereits auf die Zwischenkriegszeit zurück. An den Universitäten in den Vereinigten Staaten und an der Eidgenössischen Technischen Hochschule in Zürich haben Professoren erste «Riesenmaschinen», aber auch Programmsprachen und Software entwickelt. Deren Einsatz an Wissenschaftsinstitutionen, in der Privatwirtschaft und auch in der Verwaltung ist aber, nicht zuletzt aus Kostengründen, erst in den sechziger Jahren des letzten Jahrhunderts zur Realität geworden. ${ }^{5}$

4 Für einen allgemeinen Überblick zu den Entwicklungen vgl. RoLF H. WEBER, IT-Recht - Bausteine einer neuen Disziplin, Jusletter IT vom 23. Februar 2017.

5 Vgl. Rolf H. Weber, Information Law in Swiss Legal Culture, The Anthology of Swiss Legal Culture, Bern 2017, 7 ff. 
Hernach hat sich auch die Rechtswissenschaft die Frage gestellt, ob und wie die Computer in den Bereichen der Gesetzgebung und Rechtsanwendung einsetzbar sind. Vorerst stand das Gerät, d.h. die Hardware der Informationstechnologie, im Vordergrund; diese Konzentration auf die Hardware hat sich aber bald als zu einseitig erwiesen und es ist eine Zuwendung zur Software eingetreten.

Die engste Verbindung von Computer und Recht hat im Versuch bestanden, den Computer zu instrumentalisieren und ihn als Handwerkszeug für die Rechtswissenschaft einzusetzen. Auf diese Zeit geht auch die Gründung der Zeitschrift "Computer \& Recht» als der ältesten Zeitschrift zum IT-Recht im deutschsprachigen Bereich zurück (1982). Hauptträger der damaligen Entwicklungen sind zwei deutsche Professoren gewesen, die neben dem Recht auch eine Ausbildung in Mathematik bzw. in Psychologie hatten, nämlich Herbert FIEDLER und WilHelm SteINMÜLLER. Ihre interdisziplinär angelegten Schriften sind der Frage nachgegangen, inwieweit und in welcher Art die Technik des Rechtsanwendungsprozesses für den Computereinsatz geeignet gemacht werden könnte. ${ }^{6}$

Da die Computersprache grundsätzlich «nur» Ja/Nein-Antworten erlaubt, ist die Software so auszugestalten, dass Entscheid-Strukturen in dieser bipolaren Weise möglich sind; wie der mit der IRIS während Jahren verbundene und an der IRIS auch geehrte HERBERT FIEDLER diagnostizierte, würde der Computer im Idealfall gestützt auf den eingegebenen Sachverhalt die Rechtslage eruieren oder aus einem allgemeinen Rechtsgrundsatz eine Lebenssituation ableiten. ${ }^{7}$ Anlässlich eines von THOMAS HOEREN im März 2008 in Greifswald durchgeführten grossen Symposiums haben sich FIEDLER und STEINMÜLLER im Sinne von phänomenologischen Erinnerungen zu ihren früheren Erfahrungen geäussert; die entsprechenden Protokolle wurden hernach aber leider nicht publiziert.

WEBER (Fn. 4), Rz. 9.

Vgl. Herbert FiedLeR/Thomas Bartel/Gerhard Voogd, Untersuchungen zur Formalisierung im Recht als Beitrag zur Grundlagenforschung juristischer Datenverarbeitung, Opladen 1984; WILHELM STEINMÜLLER, Informationstechnologie und Gesellschaft, Darmstadt 1993. 


\section{Frühe Weiterentwicklungen}

Die Begrifflichkeit von «Computer und Recht» bzw. der «Rechtsinformatik» zeigt deutlich, dass eine Kombination von Technik und Recht bzw. eine Bezugnahme des Rechts auf die Technik in Frage steht. Der intellektuelle Hintergrund der Rechtsinformatik beruht somit auf der Idee, durch Computerprogramme die Rechtsanwendung zu automatisieren. Ganz offensichtlich war eine solche Betrachtungsweise zu Beginn an den Universitäten in der durch die juristische Denkweise und Dogmatik geprägten Rechtswelt ein Fremdkörper. Zwar hat das interdisziplinäre Fach «Rechtsinformatik» in den Folgejahren einen gewissen Platz in den Lehrplänen von Universitäten erhalten, ohne aber in der Ausbildung letztlich eine sehr grosse Tragweite zu erreichen. ${ }^{8}$

Einen wesentlichen Beitrag zur Fortentwicklung nach der Grundlegung der «Rechtsinformatik» hat ERICH SCHWEIGHOFER in den neunziger Jahren des letzten Jahrhunderts geleistet. Seine frühen Beschäftigungsfelder und Publikationen haben die Information Retrieval-Systeme sowie die automatische Textanalyse betroffen; mit diesen Arbeiten hat der Jubilar eine Vorreiterrolle in der Rechtsinformation eingenommen.

Ein Information Retrieval-System befasst sich mit der Repräsentation, Speicherung und Organisation von Informationen und dem Zugriff zu Informationen. ${ }^{9}$ Der Prozess des Auffindens und der Verwendung der Information durch Informations- und Kommunikationsprozesse wird durch das Information Retrieval-System, d.h. den Einsatz von Suchmaschinen, wesentlich erleichtert. ${ }^{10}$ Aufgrund der Autoritätswirkung von juristischen Informationssystemen sowie der hohen Kosten konventioneller Recherchen sind die Rechtsdatenbanken zu einem wichtigen Forschungsobjekt geworden, ${ }^{11}$ wie der Erfolg der von der Universität Wien im Wintersemester 1995/1996 angenommenen Dissertation von ERICH SchweIGHOFER, in der die wichtigsten Datenbanken im EU- und EWR-Recht detailliert untersucht werden, zeigt. ${ }^{12}$ Parallel dazu ist im Sinne

8 WEBER (Fn. 4), Rz. 10.

9 SCHWEighofer (Fn. 2), 43 m.w.Verw.

10 ERICH SchweighofER, Transparenzfiktion und Big Data, Jusletter IT vom 23. Februar 2017, Rz. 23.

11 SCHWEIGHOFER (Fn. 2), 45.

12 SCHWEIGHOFER (Fn. 1), $33 \mathrm{ff}$. 
der vorerwähnten neuen Rechtsinformatik-Disziplin das Buch «Juristische Informationsverarbeitung» erschienen. 13

Der Jubilar wies (ebenfalls) in seiner Promotionsschrift bereits darauf hin, dass durch Rechtsinformationsysteme der Zugang zu den Rechtsmaterialien zwar wesentlich verbessert werden könne, ihm aber weiterhin eine ungenügende Reduktion der Materialfülle entgegenstehe. ${ }^{14}$ Eine Strukturierung des Wissens und eine Selektionierung der Informationsflut sind bei der zunehmenden Grösse der Rechtsdatenbanken unverzichtbar. ${ }^{15}$ Um die Systeme intelligenter zu gestalten und um einen «Bau von Brücken zwischen der Artificial Intelligence und den Retrieval-Systemen» zu ermöglichen, ist die Rechtssprache als wesentliches gemeinsames Element zu analysieren. ${ }^{16}$ Diesem Ziel verschrieb sich das von ERICH SCHWEIGHOFER geleitete Forschungsprojekt «KONTERM (kontext-related terms)», das die automatischen Extrahierungen von juristischem Strukturwissen mit statistischen Methoden verfolgte. ${ }^{17}$ Mit KONTERM konnte ein erster Schritt zu einer umfassenden Wissensrepräsentation für grosse Textmengen gesetzt werden.

\section{Paradigmenwechsel in den letzten 25 Jahren}

\section{Veränderungen durch neue Technologien}

Mit der Entwicklung des Internet und v.a. der Kommerzialisierung durch das World Wide Web (1991) hat die traditionelle Rechtsinformatik eine neue Entwicklungsstufe erreicht. Die Digitalisierung in all ihren Facetten und besonderen Ausdrucksformen (Daten in Form von Bits) ist ursächlich für die stark ansteigenden Informationsflüsse geworden. Die Information selber hat zudem den Charakter eines wichtigen Gegenstands der Wirtschaft und damit eines Objekts von Vertragsbeziehungen erhalten: Die Information drückt sich in Daten aus und Daten stellen ein handelbares Gut dar.

ERICH SCHWEIGHOFER, Juristische Informationsverarbeitung, Wien 1995 (2. Aufl. Wien 1996).

SCHWEIGHOFER (Fn. 1), 117.

SCHWEIGHOFER (Fn. 1), 118.

SCHWEIGHOFER (Fn. 1), 119.

SCHWEIGHOFER (Fn. 1), 120. 
Der wirtschaftliche Bedeutungszuwachs der Information hat zu der Erkenntnis geführt, dass sich das Recht ebenfalls neu ausrichten muss, denn die Information passt (genauso wie die Technik) nicht zwingend in die traditionellen Klassifikationen des Rechts, sondern stellt eine Querschnittsmaterie dar. ${ }^{18}$ Information spielt eine Rolle in praktisch allen Rechtsgebieten, wenn zwar teilweise in unterschiedlicher Ausprägung. Insbesondere führt aber der intensivierte grenzüberschreitende Datenfluss zu neuen Problemstellungen.

Zugleich hat die traditionelle Hardware der Grossrechner-Technologie an Bedeutung verloren. Geräte werden immer kleiner und handlicher. Netzinfrastrukturen lösen sich von den traditionellen Fernmeldeanbietern und die Preise für Kommunikationsleistungen sind erheblich gesunken. Dadurch lässt sich die Informationstechnologie vielfältiger auch für weitere Teile der Bevölkerung einsetzen; ein wichtiges praktisches Beispiel ist das von ERICH SCHWEIGHOFER im Rahmen der IRIS-Symposien oft thematisierte Internet of Things. ${ }^{19}$

\section{Erweiterungen der inhaltlichen Themenpalette}

Die modernen Technologien haben dazu beigetragen, den Blick für neue rechtstheoretische Konzepte zu öffnen. Bedeutung erlangt haben insbesondere das Konzept und die Strukturierung des Informationsrechts als Querschnittsmaterie; die Lehre hat sich darum bemüht, interdisziplinäre Erkenntnisse für das Informationsrecht fruchtbar zu machen, insbesondere gestützt auf die wissenschaftlichen und praxisbezogenen Überlegungen zur Strukturationstheorie (ANTHONY GIDDENS) ${ }^{20}$ und zur Steuerungstheorie (NikLAS LUHMANN, RENATE MAYNTZ) ${ }^{21}$ als «Ordnungshilfen» im Informationsrecht.

18 Für einen Überblick vgl. URS GASSER, What is Information Law - and what could it be?, in: Gasser (Hrsg.), Informationsrecht in «e»-Umgebungen, Baden-Baden/Zürich 2002, 7-24.

19 So ist insbesondere das IRIS-Symposium 2019 dem Thema «Internet of Things» gewidmet gewesen.

20 Anthony Giddens, Die Konstitution der Gesellschaft, Grundzüge einer Theorie der Strukturierung, Frankfurt a.M./New York 1997.

21 NikLas LuHmann, Steuerung durch Recht? Einige klarstellende Bemerkungen, Zeitschrift für Rechtssoziologie 1991, 142 ff.; Renate Mayntz, Politische Steuerung und politische Steuerungsprobleme - Anmerkungen zu einem theoretischen Paradigma, Jahrbuch zur Staats- und Verwaltungswissenschaft 1987, $89 \mathrm{ff}$. 
Ausgangspunkt ist dabei die Strukturierung in systematischen Abläufen, d.h. prozedural sollten informationelle Sachverhalte und Vorgänge angemessene akteurzentrierte Vorgehensweisen ermöglichen, die verfahrensmässig die Teilnahme an der Informationsgesellschaft steuern (ROLF H. WEBER). ${ }^{22}$ Das Informationsrecht muss sich deshalb im Fadenkreuz von Gestaltung, Stabilisierung und Selbstreflexion verstärkt als institutionalisierter (und wissenschaftsbezogen interdisziplinärer) Prozess der strukturellen Kopplung zwischen den betroffenen Akteuren verstehen. ${ }^{23}$

Ein besonderes Augenmerk ist auf den Zugang und die Verwendung von Informationen zu legen. Aus diesem Grunde hat auch die Verwaltungsinformatik bzw. das E-Government an Bedeutung gewonnen: Der Einzug der Informationstechnologie in die öffentliche Verwaltung verändert Struktur und Verfahrenselemente. Im Rahmen der IRIS sind diese neuen Phänomene von ERICH SCHWEIGHOFER schon früh, nämlich im Jahre 2002 mit der Thematik «IT in Recht und Staat» ${ }^{24}$ sowie im Jahre 2003 mit dem Spannungsfeld von Rechtstheorie und E-Government, ${ }^{25}$ zur Debatte gestellt worden.

In neuerer Zeit spielen auch Open Government Data eine immer grössere Rolle; ${ }^{26}$ die Zugänglichkeit behördlicher Datenbestände ist Ausdruck eines von der Gesellschaft getriebenen Bedürfnisses nach staatlicher Transparenz. ${ }^{27}$ Es erweist sich als, wie ERICH SCHWEIGHOFER festhält, «[i]m Sinne einer demokratischen und offenen Gesellschaft [...] notwendig, dass möglichst alles Wissen transparent ist, d.h. für alle verfügbar, ohne wesentlichen zusätzlichen Aufwand nutzbar und natürlich auch für alle verständ-

RoLF H. WeBER, Strukturations- und Steuerungstheorie als «Ordnungshilfen» im Informationsrecht, Rechtstheorie 40 (2009), 516 ff. WEBER (Fn. 22), $530 \mathrm{ff}$. ERICH SchWEIGHOFER (Hrsg.), IT in Recht und Staat, Aktuelle Fragen der Rechtsinformatik 2002, Wien 2002. ERICH SchweighOFER (Hrsg.), Zwischen Rechtstheorie and e-Government, Aktuelle Fragen der Rechtsinformatik 2003, Wien 2003. Vgl. Rolf H. Weber/Christian LauX/Dominic Oertly, Datenpolitik als Rechtsthema. Agenda für Open Government Data, Zürich 2016.

27 Vgl. auch Michael SAchs/Peter ParyceK, Open government, Jusletter IT vom 1. September 2010, Rz. 6. 
lich».28 Die von der Verwaltung erhobenen und gesammelten Daten, wie beispielsweise Statistik-, Umwelt- oder Wetterdaten, sollen von Interessierten weiterverarbeitet und neu genutzt werden können. Im Zeitalter von Big Data sind indessen noch einige Schritte zu unternehmen, um echte Transparenz zu erreichen. ${ }^{29}$

\section{LEGAL INFORMATICS ALS KONSEQUENZ DES NEUEN TECHNOLOGIESCHUBS}

\section{Knowhow-Management und -Sharing in der Rechtsberatung}

Das neue technologische Umfeld trägt nicht nur zu einer effizienteren Gestaltung bisheriger Geschäftsmodelle bei, sondern vermag auch zu stark veränderten Rahmenbedingungen für die Erbringung von Dienstleistungen zu führen. Im Vordergrund stehen dabei die Veränderungen durch wertgenerierende Informationstechnologien, die sich in Aspekten wie Knowhow-Management und Knowhow-Sharing, intelligente Dokumentenanalyse, automatisierte Daten-Analyse sowie Visibilität der Angebote ausdrücken. Als Elemente im Kontext von Legal Informatics lassen sich nennen: (i) Einsatz von algorithmischer Software bei der Rechtsausübung (künstliche Intelligenz, Machine Learning); (ii) Ausbau der Technologien, welche den Umgang mit oder den Zugang zu Recht automatisieren, erleichtern, oder erheblich verändern; (iii) Verwendung von Datenverarbeitungsmethoden (inkl. Software- und Online-Dienste), welche das Rechtssystem, die Rechtsberatung, die Rechtsdienste und die juristischen Arbeitsprozesse unterstützen oder ganz automatisch durchführen. ${ }^{30}$ Legal Informatics zeichnet sich deshalb durch eine zunehmende Interdisziplinarität aus; eine kontinuierliche Verbesserung der Ergebnisse ist im Falle des Einsatzes von Algorithmen bzw. von künstlicher Intelligenz erreichbar. Vollständig neu sind diese Entwicklungen indessen nicht. Schon seit einiger Zeit wird prognostiziert, dass gewisse rechtliche Dienstleistungen keiner individualisierten Erledigung mehr bedürfen, sondern als Commodities einem Angebot in Warenhäusern ähnlich sind.

SCHWEIGHOFER (Fn. 10), Rz. 4.

SChWEIGHOFER (Fn. 10), passim.

Vgl. auch WEBER (Fn. 3), 8 f. m.w.Verw. 
Nachfolgend soll ein besonderer Bereich von Legal Informatics, der vom Technologieschub besonders betroffen ist und neue Formen des Knowhow-Management und Knowhow-Sharing erforderlich macht, etwas genauer erläutert werden, nämlich die Rechtsberatung. Die Erbringung von Rechtsberatungsleistungen ist ein Beispiel, das die Entwicklungen von der traditionellen Berufsausübung zur künftigen Leistungserbringung besonders deutlich zeigt. Rechtsberater müssen künftig einerseits in preisgünstiger Weise die Commodity-Produkte und andererseits zu individuellen Tarifen die besonderen, auf die Nachfragerseite zugeschnittenen Dienstleistungen anbieten. Denkbar ist insoweit die Erweiterung von Geschäftsmodellen in Form der Einrichtung von Plattformen; der Zugang kann offen (Information für jedermann, etwa über Rechtsentwicklungen im Ausland) oder spezifisch geschützt (z.B. nur zuhanden bestimmter Personen) sein.

Das neue Umfeld für Rechtsberatungs-Dienstleistungen lässt sich grafisch wie folgt darstellen: 31

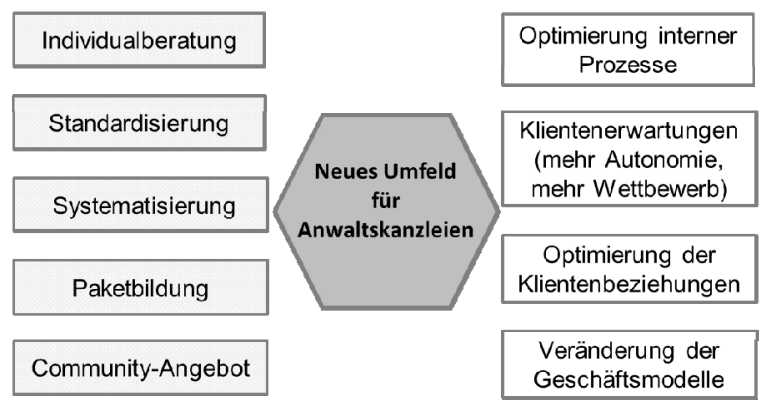

Das informationstechnologische Umfeld für die Rechtsberatungs-Dienstleistungen beeinflusst nicht nur die Strategien bei der Ausgestaltung der Geschäftsmodelle (i) im Sinne einer Veränderung in die Richtung von vertieften «Service»-Komponenten und ggf. auch (ii) im Sinne einer Erweiterung durch die Schaffung von Plattformen, sondern verlangt auch neue technische Umgebungen (z.B. «intelligente» Dokumentenanalyse, automatisierte Daten-Review, Visibilität der angebotenen Produkte), die zu- 
sätzliche Sicherheitsanforderungen nach sich ziehen. Abgesehen von der Datensammlung und der Datenanalyse geht es zudem um die Datentransformation, die jeweils sachgerechte Datenanwendung sowie die Datennachbearbeitung. Auch ERICH SCHWEIGHOFER sprach sich mit Bezug auf den Bereich der Unterstützung der Rechtsberatung und Rechtsanwendung bereits im Jahr 2007 für den Einsatz intelligenter Such- und Analysemethoden im Recht aus, da diese eine frühere und bessere Unterstützung juristischer Arbeit versprechen als die in der Forschung zur künstlichen Intelligenz dominierenden wissensbasierten Systeme. ${ }^{32}$ Solche Systeme sehen sich nämlich mit zwei Problemstellungen konfrontiert, für die zwar umfangreiche Ideen und Prototypen bestehen, ohne dass sich aber Standards herausgebildet hätten: Die intelligente Fähigkeit - im Recht «Subsumption» genannt - bedarf (i) einer klaren Repräsentation der Normen sowie (ii) einer ausreichenden Beschreibung der Fälle. ${ }^{33}$

Die einzelnen Tätigkeitsschritte bei der Rechtsberatung lassen sich in einem Vergleich zwischen dem heutigen Zustand und den künftigen Anforderungen wie folgt charakterisieren: ${ }^{34}$

- Information: Von der Recherche und dem Aktenstudium zur Datenanalyse und zum Einsatz von Algorithmen;

- Lösungssuche: Von der Beratung, gegebenenfalls durch ein Spezialistenteam, zur Erarbeitung eines (zum Teil auch interdisziplinären) Lösungsdesigns;

- Themenbearbeitung: Von der rechtlichen Ausarbeitung und dem Projektmanagement zur automatisierten Rechtsanalyse (inkl. Compliance, Due Diligence) und zur zeitlich/örtlich flexiblen Prozessorientierung;

- Präsentation der Lösung: Von der Qualitätseinschätzung, beruhend auf Vertrauen und Erfahrung, zur Vorlage objektivierter Messbarkeitsfaktoren.

32 ERICH SCHWEIGHOFER, «Intelligent Reader» statt «Intelligent Reasoner», Fortgeschrittenes Information Retrieval oder künstliche Intelligenz im Recht?, Jusletter IT vom 22. Februar 2007, passim.

33 SCHWEIGHOFER (Fn. 32), 282.

34 Vgl. WeBER (Fn. 3), 11. 
Um entsprechende neue Rechtsberatungs-Dienstleistungen anbieten zu können, erweist es sich als unumgänglich, nicht nur mit dem Recht, sondern ebenso sehr mit der Technik vertraut zu sein.

\section{Zugang zu und «Verwaltung» von Wissen}

Die neuen Technologien und die Digitalisierung erleichtern - wie erwähnt - den Informationsaustausch. Die Kontrolle über Daten ermöglicht aber auch die Verhinderung des Zugangs zu und den anschliessenden Austausch von Informationen. Als problematisch erweist sich insbesondere die faktische Privatisierung von Wissen. ${ }^{35}$ Eine solche Privatisierung kann auf einer rechtlichen oder einer faktischen Grundlage beruhen.

Weil Daten und Informationen nicht körperlicher Natur sind, vermag das «traditionelle» Eigentumsrecht des Zivilrechts keine Exklusivpositionen zu begründen, ebenso wenig wie das Immaterialgüterrecht (insbesondere das Urheberrecht) zumindest in einer kontinentaleuropäischen Perspektive. ${ }^{36}$ Wer die Verfügungsmacht über Daten hat, vermag sich deshalb in der Regel nicht auf einen Rechtstitel abzustützen, ausser wenn eine spezifische gesetzliche Schutznorm zur Anwendung kommt.

Viel bedeutsamer in der Informationsgesellschaft ist die faktische Kontrolle über Daten und damit über Informationen. Die Sammlung und Bearbeitung von Daten in proprietären Silos führt zu einer faktischen Dateninhaberschaft; derjenige, der die Kontrolle über die Daten hat, vermag Dritte von deren Gebrauch auszuschliessen. Wichtiger als die Frage des Dateneigentums ist deshalb das Problem des Datenzugangs und gegebenenfalls der Datenportabilität. ${ }^{37}$

Vgl. Willi Egloff, Der Zugang zu Fachinformationen - zwischen gesellschaftlicher Notwendigkeit und privatem Verwertungsinteresse, in: Garstka/Coy (Hrsg.), Wovon - für wen - wozu: Systemdenken wider die Diktatur der Daten, Berlin 2014, 349, 353. Im Einzelnen dazu Rolf H. Weber/Florent Thouvenin, Dateneigentum und Datenzugangsrechte - Bausteine der Informationsgesellschaft?, ZSR 2018 I, $43 \mathrm{ff}$. Im Einzelnen dazu Florent Thouvenin /Rolf H. WEBER/AlFred FrüH, Elemente einer Datenpolitik, Zürich 2020, $2 \mathrm{f}$. 
Soweit es sich um Personendaten handelt, besteht regelmässig ein Auskunftsrecht der betroffenen Person (Art. 15 DSGVO; Art. 8 DSG). Voraussetzungen und Ausmass des Auskunftsrechts mögen unterschiedlich ausgestaltet und damit Gegenstand von Auseinandersetzungen sein; im Grundsatz ist indessen der Datenzugang gewährleistet. Schwieriger ist hingegen die Rechtslage bei den Sachdaten: Soweit der Gesetzgeber keine sektorspezifische Regelung (z.B. in der Europäischen Union die Payment Service Directive 2 oder die REACH-Verordnung) vorgesehen hat, bleibt nur die Anwendung des umständlichen und in der Regel wenig erfolgversprechenden Kartellrechts. ${ }^{38}$

Weiter von praktischer Bedeutung ist die «Verwaltung» von Wissen. Mit diesem Thema befasste sich ERICH SCHWEIGHOFER beispielsweise in seinem Beitrag zur IRIS 2002 unter dem Titel «Von der Wissensrepräsentation zum Wissensmanagement im eGovernment». ${ }^{39}$ Das Wissensmanagement bezweckt optimierte Informationsprozesse und klare Aussagen über sicheres Wissen. ${ }^{40}$ Aus rechtlicher Sicht führt es eine neue Komponente für die Behandlung von Information ein, nämlich die Bewirtschaftung des Wissensflusses. «Damit werden», wie ERICH SCHWEIGHOFER in diesem Beitrag festhält, «die - neben der Information und Dokumentation - bisherigen Schwerpunkte des Datenschutzes (Geheimhaltung von personenbezogenen Informationen) bzw. Immaterialgüterrechts (ausschliessliche Nutzungsrechte von Informationen) entsprechend ergänzt. Wissen wird als knappe Ressource gesehen und muss daher einem Wissensmanagement unterworfen werden.»41

\section{Künstliche Intelligenz}

Die neueren technologischen Entwicklungen betreffen insbesondere selbstlernende Software. Die gebräuchlichen Stichworte lauten Algorithmen, künstliche Intelligenz (artificial intelligence) oder Machine Learning. Die rechtliche Herausforderung, die sich hinter diesen neuen Technologien verbirgt, ist in der Tatsache zu sehen, dass an

Dazu Thouvenin/WeBER/FrÜH (Fn. 37), 126 ff.

ERICH SCHWEIGHOFER, Von der Wissensrepräsentation zum Wissensmanagement im eGovernment, Jusletter IT vom 21. Februar 2002, Auszug aus Tagungsband IRIS-Symposium 2002, $85 \mathrm{ff}$.

SCHWEIGHOFER (Fn. 39), 88.

SCHWEIGHOFER (Fn. 39), 89. 
sich von Menschen zu fällende Entscheidungen vermehrt an Maschinen bzw. automatisierte Datenauswertungsanlagen übertragen werden (können). ${ }^{42}$ Lernende Systeme oder künstliche neuronale Netze ermöglichen algorithmische (Einzel-)Entscheidungen, die nicht ohne weiteres in die geltende Rechtsordnung einzufügen sind, weil der Grundsatz, dass die Leitplanken für das menschliche Verhalten und die gesellschaftliche Organisation durch das Recht festgelegt werden, ins Wanken gerät. ${ }^{43}$

Das Verhältnis zwischen den Menschen und den Maschinen lässt sich rechtlich in verschiedener Weise regeln. ${ }^{44}$ Fehlt es an ausdrücklichen Anordnungen, wie dies heute oft der Fall ist, sind immerhin die allgemeinen Rechtsprinzipien anzuwenden, d.h. die Verwendung von Algorithmen hat im Einklang mit der geltenden Rechtsordnung zu erfolgen. Die meisten kontinentaleuropäischen Verfassungen enthalten den Grundsatz der Menschenwürde, der unantastbar ist und vor Eingriffen in die physische oder psychische Integrität des Einzelnen schützt. Nach der Lehre der sog. «Objektformel» darf die Beurteilung der Gefährdung der Menschenwürde durch algorithmische Entscheidungen nicht davon abhängen, ob in einer abstrakten Beurteilung die automatisierten Vorgänge und Entscheidungen besser oder weniger gut sind als die menschlichen Interventionen; vielmehr kommt es «nur», aber immerhin auf die potentielle Beeinträchtigung der Menschenwürde an. ${ }^{45}$

Neben der Menschenwürde haben Algorithmen und künstliche Intelligenz weitere verfassungsrechtliche Grundsätze zu beachten, insbesondere das Gleichbehandlungsgebot bzw. das Diskriminierungsverbot, weil das Risiko besteht, dass es beim Einsatz selbstlernender Software zu Fehlentwicklungen zu Lasten von potentiell betroffenen Personen kommen kann, weil die von intelligenten Systemen verwendeten Daten nicht repräsentativ sind oder keinen relevanten Bezug zur konkreten Datenauswertung aufweisen. Weiter zu beachten ist das Legalitätsprinzip. Im Datenschutzrecht

42

WEBER (Fn. 3), 16.

Grundüberlegungen zu dieser Problemstellung sind enthalten in RoLF H. WEBER, Dürfen Maschinen über Menschen entscheiden? Eine rechtliche Auslegeordnung im Lichte neuer Technologien, Schweizer Monat, Februar 2019, 72 ff.

Christian DJEFFaL, Künstliche Intelligenz in der öffentlichen Verwaltung, Berichte des NEGZ, Nr. 3, Berlin 2018, 17 f.

Weber (Fn. 3), 17. 
hat Art. 22 DSGVO spezifische Schutzvorkehrungen bei automatisierten Datenverarbeitungen eingeführt. 46

Auch im Bereich der künstlichen Intelligenz ist es Aufgabe des Rechts, einerseits den technischen Fortschritt zu fördern und andererseits dieser Forderung aber nur insoweit $\mathrm{zu}$ entsprechen, als der Schutz von Allgemeininteressen und Dritter einem solchen Anliegen nicht entgegensteht. 47 Diese allgemeingültige Aussage machte ERICH SCHWEIGHOFER im Jahr 2001 mit Bezug auf autonome Roboter und intelligente Softwareagenten. Bereits in seinem damaligen Beitrag zum IRIS-Symposium sprach er weiter die rechtliche Erfassung von «intelligenten Maschinen» an und kürzlich ist er wieder auf seinen Gedankenansatz zurückgekommen: Vor dem Hintergrund, dass der Mensch zunehmend von Maschinen umgeben ist, die sich intelligent auf ihre Umgebung einstellen und ihm Arbeiten abnehmen, wäre zu überlegen, dass man Maschinen eine spezielle Rechtspersönlichkeit gibt, vergleichbar mit jener von Sklaven im römischen Reich. ${ }^{48}$ Der Gedankenansatz, den Maschinen die Eigenschaften von Rechtspersonen zu gewähren, wird derzeit nur von wenigen anderen Autoren geteilt. ${ }^{9}$ Überwiegend geht die Lehre davon aus, dass den Maschinen keine Rechtspersönlichkeit zukomme. ${ }^{50}$ Diese Einschätzung mag auch mit der Tatsache zusammenhängen, dass es sich bei der künstlichen Intelligenz, die algorithmische Entscheidungen ermöglicht, um eine von der Technikgestaltung abhängige und sich deshalb ständig ändernde Materie handelt. ${ }^{51}$

Weiter ist zu berücksichtigen, dass die Quantität der an Maschinen delegierten Entscheidungen für die Beurteilung der Rechtspersönlichkeit bzw. der Rechtssubjekteigenschaft ebenso wenig ausschlaggebend sein darf wie die Qualität der automatisierten Entscheidungen, die im Lichte immer ausgeklügelterer Technologien mittel-

StePhANIE HÄNOLD, Profiling and Automated Decision-Making: Legal Implications and Shortcomings, in: Corrales et al. (Hrsg.), Robotics, AI and the Future of Law, Singapore 2018, $123 \mathrm{ff}$.

47 ERICH SCHWEIGHOFER, Vorüberlegungen zu künstlichen Personen: Autonome Roboter und intelligente Softwareagenten, Jusletter IT vom 15. Februar 2001, 47.

48 ERich Schweighofer/Friedrich Lachmayer, Trends und Communities der Rechtsinformatik, Jusletter IT vom 23. Februar 2017, Rz. 20, mit Hinweis auf SchweIGHofER (Fn. 47), 45-54.

49 Gunther Teubner, Digitale Rechtssubjekte?, AcP 2018, 155, $160 \mathrm{ff}$.

50 SuSANNE BECK, Der rechtliche Statuns autonomer Maschinen, AJP 2017, 183, 186 ff.

51 WEBER (Fn. 3), 19. 
fristig besser werden sollte. Vielmehr geht es um eine rechtliche Grundsatzfrage, die in ihrer ganzen Tragweite zu beurteilen ist. 52

Angesichts der Bedeutung der mit der künstlichen Intelligenz im Zusammenhang stehenden Rechtsfragen hat die Europäische Kommission umfangreiche Arbeiten, unterstützt durch Experten verschiedener Disziplinen, aufgenommen, um die Eckpunkte einer normativen Rahmenordnung für die künstliche Intelligenz auszuloten. Abgesehen vom interdisziplinären Miteinbezug der Ethik, der Gegenstand von Erörterungen einer Expertengruppe ist, die bereits erste Richtlinien publiziert hat, ${ }^{53}$ geht es um den Miteinbezug der Zivilgesellschaft in die Diskussionen, weshalb die Kommission ein «Algorithmic Awareness Building Project» ins Leben gerufen hat. ${ }^{44}$ Weitere Beiträge von ERICH SCHWEIGHOFER zu dieser Thematik sind im Lichte der neueren Entwicklungen nicht nur erwünscht, sondern zur Befruchtung des interdisziplinären Austausches von Informatik und Recht erforderlich.

\section{AUSBLICK}

Vor fünfzig Jahren war die «traditionelle» Rechtsinformatik noch ein (zwar schönes) Orchideenfach. Nur wenige technologisch interessierte Enthusiasten haben sich mit der Verzahnung von Informatik und Recht beschäftigt. Der Fussabdruck in der Rechtslandschaft ist deshalb vorerst bescheiden geblieben. Die Arbeiten erfolgten nicht auf einem dogmatisch gepflasterten Weg zwischen schön angelegten Beeten herrlich blühender Blumen, sondern auf einem provisorischen Pfad im Bereich wild spriessender Pflanzen. 55

Diese Situation hat sich in den letzten 25 Jahren - auch dank der motivierten Mitgestaltungsaktivitäten von ERICH SCHWEIGHOFER - grundlegend geändert. Gesprochen 
wird deshalb von einem Rechtsgebiet im Wandel.56 Eingetreten ist dabei ein eigentlicher Paradigmenwechsel, der zu vielen neuen inhaltlichen Themenbereichen geführt hat. Frische Pflanzen mit überraschenden Farben sind aus dem Boden gesprossen und wachsen in die Höhe. Als Beispiele hat dieser Beitrag das Knowhow-Management und -Sharing in der Rechtsberatung, den Zugang zu und die «Verwaltung» von Wissen sowie die künstliche Intelligenz etwas genauer angesprochen.

Legal Informatics ist damit zu einer fruchtbaren und abwechslungsreichen Magerwiese geworden. Die Botanik versteht unter einer «Mager»(-Wiese) nicht eine «dünne» oder «arme» Erscheinung, sondern die Magerwiese stellt die vollendeteste Form einer schönen Blumenwiese dar. Möge ERICH SCHWEIGHOFER in den nächsten Jahren noch viele neue Pflanzen sähen! 
